\title{
Criterios para la selección de condiciones de referencia en los ríos mediterráneos. Resultados del proyecto GUADALMED.
}

\author{
Núria Bonada ${ }^{1}$, Narcís Prat ${ }^{1}$, Antoni Munné ${ }^{1}$, Maria Rieradevall ${ }^{1}$, Javier Alba-Tercedor ${ }^{2}$, Maruxa \\ Álvarez ${ }^{3}$, Juan Avilés ${ }^{4}$, Jesús Casas ${ }^{5}$, Pablo Jáimez-Cuéllar ${ }^{2}$, Andrés Mellado ${ }^{6}$, Gabriel Moyá 7 , \\ Isabel Pardo ${ }^{3}$, Santiago Robles ${ }^{4}$, Guillem Ramón $7, \mathrm{M}^{\mathrm{a}}$ Luisa Suárez ${ }^{6}$, Manuel Toro ${ }^{4}, \mathrm{M}^{\mathrm{a}}$ Rosario \\ Vidal-Abarca $^{6}$, Soledad Vivas ${ }^{5}$ y Carmen Zamora-Muñoz ${ }^{2}$ \\ ${ }^{1}$ Departament d'Ecologia. Universitat de Barcelona. Diagonal, 645. 08028 Barcelona. \\ 2 Departamento de Biología Animal y Ecología. Universidad de Granada. Campus Universitario \\ de Fuentenueva. 18071 Granada. \\ 3 Área de Ecología. Universidad de Vigo. Campus Lagoas-Marcosende. 36200 Vigo. \\ ${ }^{4}$ CEDEX. División de Ecología de los Sistemas Acuáticos Continentales. Paseo Bajo Virgen del \\ Puerto, 3. 28005 Madrid. \\ 5 Departamento de Biología Vegetal y Ecología. Universidad de Almería. Cañada de San Urbano, \\ s/n. 04120 Almería. \\ ${ }^{6}$ Departamento de Ecología e Hidrología. Universidad de Murcia. Campus de Espinardo. 30100 \\ Murcia. \\ 7 Departament de Biologia. Universitat de les Illes Balears. Crta. Valldemosa, km. 7.5. 07071 \\ Palma de Mallorca.
}

\begin{abstract}
RESUMEN
La Directiva Marco del Agua (DMA) establece la necesidad de definir las condiciones de referencia, que sirvan de patrón del estado ecológico del resto de estaciones del mismo ecotipo. Varios son los criterios que pueden ser utilizados para la selección de las condiciones de referencia. En el proyecto GUADALMED cinco localidades por cuenca muestreada, fueron escogidas a priori según la experiencia de los expertos en cada zona, uno de los criterios contemplados en la DMA. Los resultados mostraron en muchas de las estaciones seleccionadas (un 72\%) una calidad biológica alterada (IBMWP $<100$ en todas las campañas muestreadas) y un bosque de ribera perturbado (QBR $<75$ ). A partir de estos resultados se analizó si era posible el establecimiento de una serie de criterios que ayudaran a establecer condiciones de referencia. Para ello se seleccionaron diferentes criterios como la conservación del bosque de ribera, los usos naturales de la cuenca, la naturalidad del canal fluvial, la ausencia de embalses que regularan el caudal, un hábitat del lecho adecuado y concentraciones bajas de parámetros físico-químicos, en cada uno de los puntos estudiados. Algunas estaciones cumplían todos los criterios, mientras que otras podrían fácilmente cumplirlos reubicando el punto de muestreo aguas arriba o abajo del río o mediante técnicas de restauración. Los resultados fueron comparados con la tipología mediante el Sistema B establecida en otro trabajo de este volumen. En algunos ecotipos, la falta de estaciones de referencia y la imposibilidad de encontrarlas dadas las características de la cuenca y el tramo, implica la imposibilidad de establecer condiciones de referencia y la necesidad de establecer el Máximo Potencial Ecológico. Al comparar los criterios establecidos para cada estación con los valores de los índices biológicos hallados en todas las campañas, se observa que los criterios establecidos son adecuados, ya que las estaciones que los cumplen tienen un IBMWP y QBR superior. Este trabajo pretende establecer unos criterios generales que serán utilizados como base para la selección y validación de condiciones de referencia en la segunda fase del proyecto GUADALMED.
\end{abstract}

Palabras clave: referencia, criterios, Directiva Marco del Agua, ecotipo, ríos mediterráneos

\section{ABSTRACT}

The water Frame Directive (WFD) establishes the need to define reference conditions in order to determine the ecological status of all the test sites from each ecotype. Several criteria may be used to select reference conditions. In the GUADALMED project, five localities per basin were selected a priori according to the experience of experts, which is one of 
the criteria considered in the WFD. Results showed that in $72 \%$ of selected reference conditions, water quality was poor $(I B M W P<100$ in all sampled seasons) and the riparian vegetation, altered $(Q B R<75)$. Consequently, we established a set of new criteria for appropriate selection of reference conditions for natural riparian vegetation status, natural basin uses, unaltered fluvial channel, absence of reservoirs, diverse river habitat conditions and low nutrient concentrations. A few sites conform to all these criteria, whereas others may comply when a site upstream or downstream is considered instead, or after restoration techniques are applied. Results obtained were compared with the previously established System B typologies. For some ecotypes, no reference sites were found occasionally because of the difficulty in finding given basin or river reach characteristics. In these cases, a "Maximum Ecological Potential" was used instead of the reference condition. When the criteria established were compared with the biological indices across seasons for each site, a positive and significant relationship was found between all criteria and the IBMWP and QBR indices. Here, we present a set of criteria for use as a general framework to select and validate reference condition during the second phase of the project GUADALMED.

Key words: reference, criteria, Water Frame Directive, ecotype, mediterranean rivers

\section{INTRODUCCIÓN}

Los criterios para la determinación del estado ecológico propuestos por la Directiva Marco del Agua (DMA), están basados en parámetros biológicos y son relativos a las condiciones de referencia de cada grupo de puntos o tipo de río (D.O.C.E., 2000). Según Chovarec et al. (2000) una localidad de referencia se define como el estado que ha existido antes de las perturbaciones humanas, o al menos sin influencias humanas que hayan alterado de manera significativa las características naturales de un río. Owen et al. (2001), dan una definición más precisa basada en la DMA y consideran que una estación de referencia es aquella que tiene valores físico-químicos, hidromorfológicos y biológicos correspondientes a las estaciones no perturbadas, y que además, deben tener concentraciones de contaminantes específicos sintéticos o no, cercanas a cero o al menos indetectables. Así pues, son equivalentes a las condiciones control, que se utilizan en otros trabajos (Reynoldson et al., 1997).

El concepto de condición de referencia es ampliamente conocido y utilizado para determinar la calidad ecológica de un tramo de río (Reynoldson et al., 1997). Así, en EE.UU está siendo utilizado por la EPA (US Environmental Protection Agency) (Davis \& Simon, 1995), en el Reino Unido para la clasificación de ríos
(Wright, 1995), en Australia para el "National River Health Program" (Parsons \& Norris, 1996), en Sur África para el "River Health Programme" (Eekhout et al., 1996) y en Europa se pretende usar de forma extensiva en la DMA (D.O.C.E., 2000).

La utilización de la condición de referencia, como base para la determinación de la calidad ecológica de otras localidades mediante comparación (D.O.C.E., 2000; International Standard on Water Quality, 2000), es una aproximación novedosa que difiere ampliamente de las tradicionales (Reynoldson et al., 1997) - donde la calidad de una localidad era comparada con la hallada aguas arriba de la perturbación - ya que puede referirse a varios grupos de localidades de ríos o cuencas distintas, aunque todos ellos agrupados en la misma tipología (o ecotipo) (Gibson et al., 1994; Hughes, 1995; Barbour et al., 1996). Con ello, la obtención previa de una tipología es importante para el establecimiento de las condiciones de referencia (Resh et al., 1995; Gerritsen, 1995; D.O.C.E., 2000), de tal manera que una vez conocidos los valores de las diferentes variables medidas en cada punto, la calidad del agua de esa localidad puede referirse como un \% de cambio respecto a la condición de referencia del ecotipo al que pertenece (Wrigth et al., 1984; Moss et al., 1987; Resh et al., 1995). 
En Resh et al. (1995) aparecen una serie de ideas a tener en cuenta para la selección de los puntos de referencia: 1) Deberían pertenecer dentro de la "ecoregión" de interés; 2) No deben existir alteraciones río arriba, ni presentar ningún foco contaminante, vertidos o contaminación difusa; 3) La zona circundante debe poseer una densidad de población y de infraestructuras baja, y una reducida actividad agrícola; 4) No deben existir repoblaciones piscícolas en la zona; y 5) El drenaje de la localidad debe ser a través de terrenos públicos. Además, la presencia de especies alóctonas o invasivas también deberían tenerse en cuenta (Owen et al., 2001).

Varios son los criterios y métodos que se han propuesto y aplicado para la selección de puntos de referencia (Hughes, 1995; Johnson, 1993; Reynoldson et al., 1997). Los métodos basados en muestreos extensivos son los más utilizados en Europa, a pesar de estar restringidos a una región concreta y ser costosos de inicializar (Owen et al., 2001). La opinión del experto es un método fácil si se disponen de estudios previos en la zona a muestrear, aunque debe ser validada debido al sesgo que puede presentar (Owen et al., 2001). Por otro lado, los datos históricos, los modelos predictivos o la paleolimnología también son contemplados por la DMA a pesar de que su obtención es compleja y son poco utilizados. Aunque todos los métodos poseen alguna limitación, la idea común es que un punto de referencia debe estar mínimamente perturbado (Resh et al., 1995; Gerritsen, 1995; Reynoldson et al., 1997; D.O.C.E., 2000). La DMA por su parte, propone que la determinación de los puntos de referencia debe hacerse en base a los parámetros de calidad ecológica (D.O.C.E., 2000), exigiendo una muy buena calidad de los índices en estos puntos. Además, otros métodos no contemplados, podrían ser utilizados. Así por ejemplo, en la Península Ibérica, para el estudio realizado sobre la regionalización del río Ebro se utilizaron los datos disponibles en la administración de los últimos años, método que aunque no directamente contemplado, también da buenos resultados en el caso de que se dispongan de estos datos (Munné \& Prat, 1999).

Los ríos mediterráneos del levante peninsular y Baleares, están altamente afectados por perturbaciones humanas de diferente tipo (Prat, 1993), hecho que puede limitar el establecimiento de condiciones de referencia para cada ecotipo según los criterios de la DMA, sobretodo en aquellas que se refieren a los tramos medios-bajos de los ríos. Para estos tramos, las condiciones de referencia podrían ser reconstruidas técnicamente mediante datos históricos, paleológicos o modelos (Chovarec et al., 2000; Owen et al., 2001), métodos también contemplados en la DMA. No obstante, en ausencia de todos estos datos, la referencia puede basarse en la opinión de los expertos. En último caso si las perturbaciones humanas hacen imposible el establecimiento de condiciones de referencia, se podría establecer lo que se conoce en la DMA como Máximo Potencial Ecológico, correspondiente a la calidad ecológica mejor que se le puede asignar a un ecotipo.

Los objetivos de este trabajo son: Validar mediante los índices biológicos y de ribera (IBMWP - ver Alba-Tercedor et al., este volumen-y QBR) las estaciones establecidas $a$ priori como de referencia en la primera fase del proyecto GUADALMED; establecer los criterios básicos a tener en cuenta para la selección de estaciones de referencia para la segunda fase del Proyecto; comparar los resultados obtenidos con la tipología del Sistema B y modificada por los expertos que se expone en Bonada et al., este volumen.

\section{MATERIAL Y MÉTODOS}

Dada la variabilidad de condiciones que se pueden encontrar en las diferentes ecoregiones y los ecotipos dentro de ellas, la manera de seleccionar las estaciones de referencia puede ser múltiple. De forma sintética los métodos para establecer estaciones que reflejen las condiciones de referencia se pueden agrupar en varias 
Tabla 1. Métodos para el establecimiento de las condiciones de referencia. Adaptación de los propuestos por Owen et al., 2001. Methods to establish the reference conditions. Adapted from Owen et al., 2001

\begin{tabular}{|c|c|c|}
\hline Método & Ventajas & Inconvenientes \\
\hline Opinión del experto & $\begin{array}{l}\text { Puede incorporar datos históricos, } \\
\text { opiniones y conceptos actuales }\end{array}$ & Sesgo. Subjetividad. \\
\hline Datos históricos & Poco costosos de obtener. & $\begin{array}{l}\text { Datos variables, pocos métricos. La calidad } \\
\text { de los datos puede ser pobre o desconocida. }\end{array}$ \\
\hline Datos disponibles & Fáciles de obtener. & Sesgo, datos variables. \\
\hline Paleolimnología & $\begin{array}{l}\text { Incorpora datos físico-químicos } \\
\text { y biológicos. }\end{array}$ & $\begin{array}{l}\text { Limitados a sistemas lénticos. } \\
\text { Costos iniciales elevados. }\end{array}$ \\
\hline Modelización & Específicos de cada región. & $\begin{array}{l}\text { Requiere gran cantidad de datos, calibración } \\
\text { y validación. }\end{array}$ \\
\hline Estudios & Específicos de cada región. & Costosos de inicializar. \\
\hline
\end{tabular}

categorías (Tabla 1). En el caso del proyecto GUADALMED, dado el conocimiento fragmentario que se tenía del conjunto de los ríos a estudiar, inicialmente se empleó el método de la opinión del experto. En este caso, cada uno de los expertos seleccionó las estaciones que juzgó como de referencia en su cuenca. Para ello, en las 12 cuencas muestreadas se escogieron un mínimo de 5 localidades a priori, antes del muestreo, mediante la experiencia de cada institución en su zona. Estas estaciones deberían abarcar, en la medida de lo posible, distintos tramos de ríos o condiciones. Con ello, el establecimiento de las condiciones de referencia se hizo previamente a la tipología.

Para comprobar hasta qué punto las estaciones de referencia presentaban características biológicas compatibles con una alteración baja o nula por la actividad humana, se utilizaron los índices biológicos de todas las campañas muestreadas. Como criterios de obligado cumplimiento se adoptó que una estación era buena como de referencia si el valor del IBMWP en todas las campañas era superior a 100 (AlbaTercedor \& Sánchez-Ortega, 1988) y el QBR superior a 75. Este último límite se escogió, debido a que el 95, propuesto por Munné et al. (1998), es muy exigente ya que supone un estado natural, mientras que el valor de 75 admite una mínima perturbación. Una vez conocidos los resultados se establecieron una serie de criterios para que la selección de las estaciones de referencia fuera más objetiva. Los criterios utilizados se exponen en la Tabla 2. Con ellos se construyó una matriz para todas las estaciones de SÍ/NO, donde SÍ indicaba que cumplía criterio y NO, que no lo cumplía, que fue transformada en $1 / 0$, respectivamente. Para cada punto de muestreo se obtuvo un valor correspondiente a la suma de todos los criterios de referencia, al que llamamos SCR (Anexo 1).

Debido a que en la DMA se expone que las estaciones de referencia deben establecerse para cada uno de los ecotipos, las estaciones fueron ordenadas según los ecotipos del Sistema B (ver el trabajo de Bonada et al., este volumen), y así fueron analizadas.

\section{RESULTADOS}

\section{Estaciones de referencia por criterio de expertos y calidad ecológica}

Para testar las estaciones de referencia seleccionadas a priori por los expertos, se analizaron el número de estaciones que tenían una buena calidad ecológica QBR mayor a 75, y IBMWP supe- 
Tabla 2. Criterios para la selección de las condiciones de referencia en el proyecto GUADALMED. Criteria to select reference conditions in the GUADALMED project.

$<\mathbf{1 0 \%}$ uso de la cuenca (subcuenca) urbano, agrícola o industrial

Con ello se quería evaluar la dominancia o no de una cuenca con usos naturales. La respuesta Sí indicaría cuenca sin alteraciones.

\section{Bosque de Ribera Naturalizado}

Este criterio se refería al estado de conservación de la cobertura vegetal de las riberas. Se consideraría Sí cuando la cobertura existente en un punto de muestreo es la que debería de tener para ese tipo de río. En la mayoría de los casos, la referencia será un bosque de ribera con árboles y cobertura total. No obstante, en otros casos no tiene que ser así; por ejemplo en cabeceras de más de $2000 \mathrm{~m}$ de altitud puede no existir cobertura por causas naturales y esto sería la situación de referencia. En las ramblas o en los ríos temporales el estrés hídrico puede hacer que la cobertura arbórea no exista o sea menor al $100 \%$, lo que sería la situación natural.

\section{Bosque de Ribera constituido por especies autóctonas}

Se consideraría Sí cuando el bosque de ribera estaba formada exclusivamente por especies vegetales autóctonas. En el caso de que se encontrara un solo pie alóctono y este se tratara de una especie naturalizada (Robinia pseudoacacia, por ejemplo) no se tendría en cuenta. La lista de especies no autóctonas se encuentra en la hoja de campo del QBR.

\section{Bosque de Ribera sin alteraciones}

En este caso, un punto de muestreo tendría un bosque de ribera sin alteraciones cuando no existían infraestructuras importantes en las riberas, tales como fábricas, casas, polideportivos,.... La respuesta SÍ indicaría bosque sin alteraciones.

\section{Canal fluvial natural}

Se consideraría Sí cuando el río no está canalizado ni presenta escolleras que regulen el canal del río, o presas transversales $\mathrm{u}$ otras estructuras similares.

\section{Río sin Regulación}

Un punto de muestreo sometido a una regulación por embalses aguas arriba, se consideraría regulado. La respuesta SÍ indicaría tramo sin regulación.

\section{Hábitat del lecho adecuado}

Se consideraría SÍ cuando el tramo estudiado tuviera un sustrato correspondiente al del tipo al que pertenece, es decir piedras grandes en partes altas; cantos y gravas en tramos medios y bajos y arenas o limos en las zonas aluviales.

\section{Concentración de amonio}

En este caso un SÍ significaría una concentración de amonio menor a $0.5 \mathrm{mg} / 1$.

\section{Concentración de $\mathbf{N}$-Nitritos $<\mathbf{0 . 0 1}$}

En este caso un SÍ significaría una concentración de N-nitritos menor a $0.01 \mathrm{mg} / \mathrm{l}$.

\section{Concentración de P-Fosfatos $<0.05$}

En este caso un SÍ significaría una concentración de P-fosfatos menor a $0.05 \mathrm{mg} / \mathrm{l}$.

rior a 100. Debido a que el IBMWP está calculado para las distintas campañas, para cada estación se calculó el \% de campañas con IBMWP superior a 100. Así un valor de 100\% de IBMWP indicaría que en todas las épocas muestreadas se halló una muy buena calidad del agua. Los datos obtenidos fueron analizados separadamente según si la estación fue seleccionada como de 
Tabla 3. Validación de las estaciones de referencia seleccionadas a priori por los expertos, según sus \%IBMWP y QBR. Validation of the reference sites selected a priori by the expert panel using $\% I B M W P$ and $Q B R$ indices.

\section{Referencia para el IBMWP}

REFERENCIA

Número estaciones con 100\% IBMWP >100 23

Número estaciones totales de REF 54

$\%$ estaciones de referencia buenas para el IBMWP $\quad 42.6$

\section{NO REFERENCIA}

Número estaciones con 100\% IBMWP> 100

Número estaciones totales de NO-REF 97

$\%$ estaciones de referencia buenas para el IBMWP 4.1

\section{Referencia para el QBR}

REFERENCIA

Estaciones con un QBR>75

Número estaciones totales de REF

$\%$ estaciones de referencia buenas para el QBR

NO-REFERENCIA

Estaciones con un QBR>75

Número estaciones totales de NO-REF

$\%$ estaciones de referencia buenas para el QBR

\section{Referencia para IBMWP y el QBR}

\section{REFERENCIA}

Estaciones con 100\% IBMWP>100 y QBR >75

Número estaciones totales de REF

$\%$ estaciones de referencia buenas

para el IBMWP y el QBR

\section{NO-REFERENCIA}

Estaciones con 100\% IBMWP>100 y QBR >75

Número estaciones totales de NO-REF

$\%$ estaciones de referencia buenas para el IBMWP y el QBR mientras que tan solo un $28 \%$ lo son para ambos índices. Ello indicaría que la opinión de los expertos ha fallado en un $72 \%$ de los casos. Por otra parte, solamente una estación no seleccionada como de referencia podría serlo para el IBMWP y el QBR, mientras que 9 podrían serlo para el QBR y 4 para el SBMP. Ello indicaría que a pesar de que los expertos seleccionaron algunas estaciones que no deberían serlo, las demás estaciones muestreadas tampoco tienen demasiado potencial para ello.

Según se muestra en la Tabla 4, las estaciones seleccionadas por los expertos que cumplen

Tabla 4. Estaciones de referencia seleccionadas a priori por los expertos con buena calidad ecológica para el IBMWP y el QBR, y ordenadas según su ecotipo (Bonada et al., en este volumen). Reference sites selected a priori with good ecological status according to IBMWP and QBR values of indices, grouped by ecotype (Bonada et al., in this issue).

\begin{tabular}{|c|c|}
\hline $\begin{array}{l}\text { Estaciones con } \\
\% \text { IBMWP>1 } 00 \text { y } Q B R>75\end{array}$ & $\begin{array}{l}\text { Sistema B } \\
\text { modificado expertos }\end{array}$ \\
\hline JU8-R & $\mathrm{Cal} / \mathrm{Cab}$ \\
\hline SE1-R & $\mathrm{Cal} / \mathrm{Cab}$ \\
\hline AD5-R & $\mathrm{Sil} / \mathrm{Cab}$ \\
\hline \multirow[t]{2}{*}{ B35-R } & $\mathrm{Sil} / \mathrm{Cab}$ \\
\hline & $\mathrm{Sil} / \mathrm{MB}$ \\
\hline JU2-R & $\mathrm{Cal} / \mathrm{MB}$ \\
\hline L54 & $\mathrm{Cal} / \mathrm{MB}$ \\
\hline L60a-R & $\mathrm{Cal} / \mathrm{MB}$ \\
\hline SE16-R & $\mathrm{Cal} / \mathrm{MB}$ \\
\hline \multirow[t]{3}{*}{ SE18-R } & $\mathrm{Cal} / \mathrm{MB}$ \\
\hline & $\mathrm{Cal} / \mathrm{GB}$ \\
\hline & Llan-Aluvial \\
\hline B7a-R & Temporales \\
\hline AG1-R & Karst \\
\hline AG7-R & Karst \\
\hline AL7-R & Karst \\
\hline L44-R & Karst \\
\hline PO7-R & Karst \\
\hline \multirow[t]{2}{*}{ PO8-R } & Karst \\
\hline & Ramblas \\
\hline
\end{tabular}

referencia o no. En la Tabla 3, se muestra el número de estaciones que cumplirían la referencia para el IBMWP, el QBR y ambos índices a la vez. Las estaciones con mayor potencial para cumplir la condición de referencia se definieron como aquellas que la cumplieran para el IBMWP y el QBR (como únicos índices biológicos medidos). Así pues, según los resultados, un $42.6 \%$ de estaciones consideradas de referencia lo son para el IBMWP, y un 57.4\% para el QBR, 
los criterios del IBMWP y el QBR pertenecen a distintos ecotipos del Sistema B, y en 4 de los 8 resultantes no existen estaciones de referencia. El límite establecido para el IBMWP de 100 podría ser demasiado elevado en algunos tipos (ramblas, llanura aluvial o grandes ríos calcáreos), debido a las características hidrológicas, químicas o estructurales naturales, y ello podría ser el origen de la falta de estaciones de referencia en algún ecotipo. Otro aspecto importante a tener en cuenta es la variación estacional de las comunidades de macroinvertebrados, aunque estudios realizados en algunas de las cuencas muestradas ponen de manifiesto que el IBMWP es independiente de la estacionalidad (Zamora et al., 1995).

\section{Criterios para la selección de las condiciones de referencia, de forma menos subjetiva}

En el Anexo 1, se presentan las localidades muestreadas agrupadas por ecotipo, y con el correspondiente valor de SCR. De las 54 estaciones seleccionadas a priori por los expertos, $78.6 \%$ no cumplían los criterios de referencia para serlo (tenían una SCR baja), lo que vuelve a poner de manifiesto la necesidad de reconsiderar las estaciones seleccionadas como de referencia. Del total de estaciones muestreadas, solamente 15 tienen un 10 de SCR, y por tanto se consideran como localidades que cumplen todos los criterios de referencia (Anexo 2). Del resto de estaciones, 36 poseen valores de SCR entre 7 y 9 , siendo los criterios referentes a la ribera los que están más frecuentemente alterados. Debido a que las riberas son altamente heterogéneas en el espacio (Munné et al., 2003), el valor de SCR podría mejorarse, si fuera posible, reubicando el punto de muestreo en una zona cercana a la muestreada que presentara una mejor conservación de la vegetación riparia, lo cual no siempre ocurre. Lo mismo sucede en aquellas estaciones en las que el factor limitante para la referencia es algún parámetro físico-químico, en cuyo caso debería testase su efecto sobre la fauna macroinvertebrada para establecer su potencial de referencia. De la misma manera, existen estaciones que fallan en algún parámetro físico-químico y de ribera potencialmente mejorables, como las JU8-R y MI7 con SCR de 6. En otros casos, el factor que hace disminuir es el referente al \% de la cuenca natural o al de canalización o regulación. Estos factores son considerados como altamente perturbadores de las comunidades bentónicas y prácticamente imposible de restaurar por lo que los puntos de muestreo no podrían ser considerados de referencia, a pesar de poseer un bosque de ribera y un sustrato bien estructurados. Este es el caso por ejemplo de un punto en la parte media-baja del río Llobregat que si bien fue considerado como de referencia por los expertos, su ubicación aguas abajo del embalse de la Baells, hace que se reconsidere su condición de referencia, a pesar de poseer una buena calidad biológica y riparia (Prat et al., 1999; 2000; 2001).

Cuando las estaciones con elevados SCR se contrastan con los ecotipos se observa que las cabeceras silíceas, cabeceras calcáreas, ríos temporales, karsts, tramos medios bajos de ríos calcáreos y ramblas poseen estaciones que cumplen los criterios de referencia. No obstante, el número de estaciones de elevado SCR es reducido en algunos de ellos (ramblas, por ejemplo).

Por otra parte, el ecotipo tramos bajos de ríos grandes calcáreos, no posee ninguna estación con SCR de 10, debido a que por la ubicación de este tipo, las estaciones suelen estar canalizadas y la cuenca siempre está alterada y regulada, lo que supone que el mínimo de SCR exigido en este ecotipo, si obviamos estos tres criterios, debería ser de 7. Las estaciones que en este ecotipo tuvieran una SCR de 7 o mayor (sin llegar a 10) debido a que tienen alterado sólo alguno de estos criterios, podrían ser consideradas como las potenciales de referencia dentro de ese tipo, que podrían ser utilizadas para calcular el Máximo Potencial Ecológico (MPE) del ecotipo. Según esto, dos estaciones del Júcar, cumplen estas características, aunque son insuficientes estadísticamente para establecer el MPE.

Los demás ecotipos (tramos medios bajos de ríos silíceos o los ríos de la llanura aluvial) no poseen estaciones potenciales a cumplir los cri- 
terios debido a su elevado grado de alteración antrópica y además las SCR halladas son inferiores a 7 , con lo que no se pueden establecer estaciones potenciales a cumplir criterios para el MPE. Ello implicará que para la segunda fase del proyecto GUADALMED se deberá poner más esfuerzo en seleccionar estaciones de estos ecotipos que posean una elevada SCR para que cumplan criterios de referencia o de MPE.

Una vez establecidos estos criterios, se quiso determinar si el valor de SCR (indicador de potencialidad de referencia), proporcionaba también una indicación de la calidad biológica y riparia (medidas mediante el IBMWP y el QBR, respectivamente), con el fin de testar de alguna manera la validez de los criterios seleccionados. Para ello, se presentan unos gráficos de disper- sión (Fig. 1a y 1b) donde se relacionan los valores de SCR de todas las estaciones con el correspondiente \%IBMWP>100 y el QBR. Los resultados indican que a valores elevados de SCR hay una tendencia a que las estaciones tengan valores elevados de \%IBMWP y QBR. No obstante, existen algunas estaciones que a pesar de tener una SCR elevada tienen un \%IBMWP o un $\mathrm{QBR}$ reducido, aunque las correlaciones de Pearson calculadas muestran que la tendencia es positiva y significativa $(\mathrm{r}=0.61$ para el \%IBMWP y $\mathrm{r}=0.63$ para el $\mathrm{QBR}, \mathrm{p}=0.001$ ). Las estaciones que huyen de esta tendencia podrían estar afectadas por varios factores. Referente al QBR, estas localidades podrían ser las que fácilmente cumplirían los criterios de referencia, si se reubicaran en un lugar cercano,
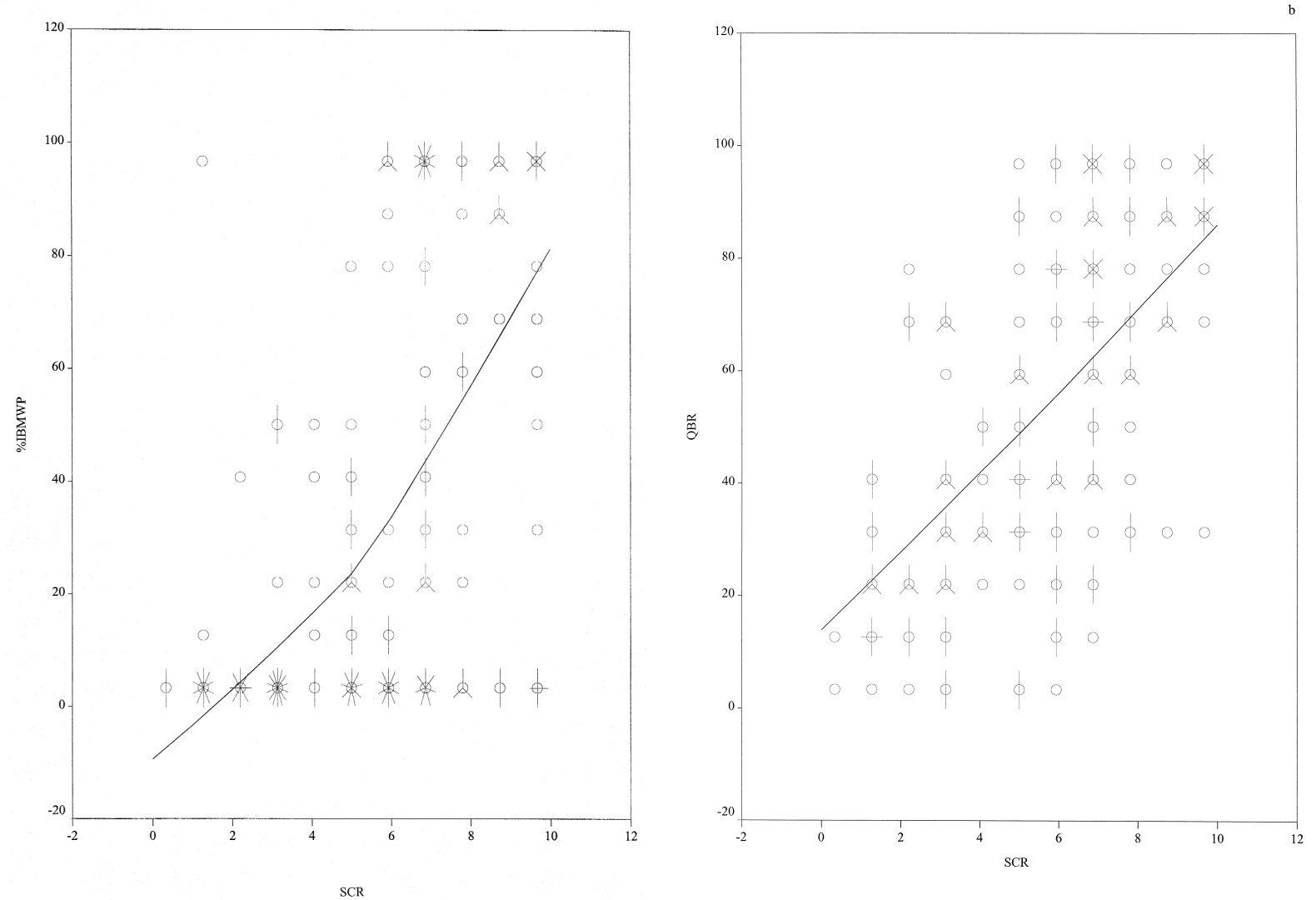

Figura 1: Relación entre la SCR (Suma de Condiciones de Referencia) y los valores de \%IBMWP (a) y QBR (b) para todas las estaciones muestreadas. El número de líneas de cada círculo indica el número de casos. Relationship between the SCR (Sum of Reference Conditions) and the values of \%IBMWP (a) and QBR (b) for all the sampling sites. The number of lines on each circle indicates number of cases. 
ya que para muchas de ellas el estado del bosque de ribera es el factor limitante. En el caso de \%IBMWP, el problema podría ser que las estaciones con SCR elevada y \%IBMWP reducido, pertenecieran a ecotipos cuyos rangos de calidad deben ser redefinidos, inclusive aquellos en los que no es posible el establecimiento de estaciones que cumplan criterios de referencia sino de MPE. A pesar de todo ello, los resultados parecen indicar que los criterios escogidos para determinar la referencia son adecuados, ya que reflejan, al mismo tiempo, la calidad biológica del sistema, según se indica en la DMA, aunque es necesaria un establecimiento de los rangos de calidad para cada ecotipo.

\section{DISCUSIÓN}

Una condición de referencia puede ser establecida utilizando varios criterios (Hughes, 1995; Johnson, 1993; Reynoldson et al., 1997), pero siempre teniendo en cuenta una serie de consideraciones (Resh et al., 1995). Algunos de estos métodos están basados en datos históricos de los tramos a estudiar, aunque a veces éstos son inaccesibles o bien están basados en métodos desconocidos y diferentes a los que se quieren aplicar (Reynoldson et al., 1997). También se han propuesto métodos basados en la paleolimnología, aunque ello supone importantes limitaciones en los ríos (Bennion \& Fluin, 2001). Estudios experimentales con sustancias tóxicas también podrían ser usados, aunque son de difícil aplicación a toda la comunidad de macroinvertebrados (Reynoldson et al., 1997), hecho que no sería muy adecuado si se pretende determinar el estado ecológico del sistema. Los métodos más utilizados para la determinación de las condiciones de referencia están basados en los valores que proporcionan los índices biológicos (Reynoldson et al., 1997), aunque se debería tener en cuenta su variabilidad anual y referente al hábitat (Barbour et al., 1999) y al ecotipo (Wrigth et al., 1984; Moss et al., 1987; Resh et al., 1995). No obstante, en algunos casos se ha utilizado el "conocimiento del experto" para determinar las estaciones de referencia, aunque éste depende de su experiencia en la zona a muestrear (Reynoldson et al., 1997).

En nuestro caso, las estaciones de referencia fueron escogidas a priori según la experiencia de cada grupo de trabajo en la zona estudiadas (e.g., Mellado et al., 2002; Prat et al., 1999, 2000, 2001; Varios Autores, 1988, Ortega et al., 1991; Vidal-Abarca et al., 1991). Como se desprende de los resultados, el criterio de los expertos falló en la asignación de una estación como de referencia para el IBMWP y el QBR ( $72 \%$ de error). Este error, podría ser debido en parte, a que se establecieron un mínimo de 5 estaciones por cuenca que deberían ser de referencia, cuando nuestros resultados han mostrado que las que podrían serlo son muchas menos, e incluso de la primera aproximación de los expertos, solamente una que no fue considerada podía haber estado.

Todo ello supuso la reconsideración de lo que debería ser la referencia, estableciendo una serie de criterios más objetivos para determinar la potencialidad de que una estación fuera de referencia. Estos nuevos criterios, suponen que el número de estaciones de referencia es menor que las establecidas por los expertos anteriormente ( 15 según los nuevos criterios respecto 54 a priori), lo que ayuda a reducir el error cometido por el experto. No obstante, aparecen algunas estaciones, que si bien no pueden ser consideradas como de referencia para la primera fase del GUADALMED, si podrían serlo en la segunda fase del Proyecto si se reubicaran en algún punto cercano con mejores condiciones, ya que los valores de SCR que tienen son relativamente elevados (entre 7 y 9, y en algunos casos 6) y tienen un buen porcentaje de cuenca natural y no están ni regulados ni canalizados. La mayor parte de estos puntos, fallan en cuanto al bosque de ribera, lo que indicaría bien la elevada degradación de los bosques de ribera de las cuencas mediterráneas estudiadas.

Los resultados de la validación de estos nuevos criterios para la referencia son positivos, en el sentido que la SCR (que indicaría el grado de 
referencia de un punto) está bien correlacionada con los valores de IBMWP y QBR. En algunos casos existen elevados valores de SCR que poseen un \%IBMWP menor de 100, lo que podría ser una consecuencia de que los criterios de selección de referencia fueran incompletos o que otros factores que no se han considerado fueran importantes, pero también a que el límite establecido de 100 para el IBMWP sea demasiado exigente en algunos de los ecotipos. Así pues, para la segunda fase del GUADALMED, se pretende incidir en el tema de la selección de referencia, poniendo especial atención en el establecimiento de rangos de calidad para cada tipo y en la validación de los criterios aquí expuestos.

Tal y como especifica la DMA el establecimiento de las condiciones de referencia debe hacerse teniendo en cuenta los distintos ecotipos hallados según el sistema A o B, y cada ecotipo debería tener un mínimo de estaciones de referencia suficiente (D.O.C.E., 2000). Según los resultados mostrados en Bonada et al. (este volumen), una tipología mediante el sistema A supone el establecimiento de demasiados ecotipos como para encontrar diferencias en la composición de macroinvertebrados y estaciones de referencia de cada ecotipo (16 grupos o tipos), en cambio la tipología obtenida con el sistema $\mathrm{B}$, parece mejor desde el punto de vista ecológico y de simplificación de la información. Según esta tipología aparecen 9 ecotipos, pero los resultados muestran que no todos poseen estaciones que cumplan o pudieran cumplir los criterios de referencia, lo que dificulta el establecimiento de los límites de calidad para cada tipo. Así, para los ecotipos tramos medios bajos de cuencas silíceas, tramos bajos de ríos grandes en cuencas calcáreas y la llanura aluvial se debería buscar para la segunda fase del Proyecto localidades potenciales a cumplir estos criterios. En el caso de que no fuera posible, debido a la elevada alteración de estos ecotipos, se podrían establecer para cada ecotipo el Máximo Potencial Ecológico, siguiendo la denominación de la DMA. En estos casos, en los que no existen estaciones de referencia posibles para un ecotipo, la DMA propone el uso de modeliza- ción del potencial que debería tener esta ecoregión, mediante modelos predictivos tipo RIVPACS (Wrigth, 1995), datos históricos o paleolimnológicos (D.O.C.E., 2000). No obstante, debido a la inexistencia de estos datos en la mayoría de cuencas mediterráneas peninsulares y baleáricas, y a que en su caso, la metodología pueda ser distinta a la utilizada para el proyecto GUADALMED, la modificación de los rangos de calidad para cada ecoregión, sería una alternativa, estableciendo unos mínimos (potencial ecológico) para cada tipo.

Debido a que los distintos países miembros utilizan diferentes elementos para la determinación del estado ecológico (Johnson, 2001), los métodos usados para el establecimiento de las condiciones de referencia también varían (Owen et al., 2001). Así, los resultados de muestreos extensivos de datos físico-químicos, hidromorfológicos y macroinvertebrados son los más comunes. Según Owen et al. (2001), la opinión del experto debería ser el último método a utilizar debido al enorme sesgo que puede presentar, y debería ser validado, tal y como se ha demostrado en este trabajo. No obstante, este método juntamente con los datos históricos también están siendo ampliamente extendido, seguidos de los modelos predictivos y la paleolimnología. Estos tres últimos métodos son complejos, ya sea porque en el caso de los datos históricos se desconoce la metodología usada para su obtención (Reynoldson et al., 1997) o no se midieron todas las variables necesarias, o bien porque no son directamente aplicables en ríos (paleolimnología) (Owen et al., 2001). En el caso de los métodos predictivos, los tipo RIVPACS (Wrigth, 1995), BEAST (Reynoldson et al., 1995) o AusRivAS (Parsons \& Norris, 1996) han sido utilizados en países con una base de datos previa muy amplia y diseñada para este uso.

El uso de un PRECE (Protocolos Rápidos de Evaluación de la Calidad Ecológica, ver JáimezCuéllar en este volumen) implica una simplificación en la toma de muestras y procesado (Resh et al., 1995; Barbour \& Gerritsen, 1996) para aumentar la rapidez pero perdiendo el 
mínimo de información. Ello supone que el uso de réplicas en cada uno de los puntos es limitado o inexistente, lo que implica la imposibilidad de aplicar métodos estadísticos paramétricos. No obstante, varios autores han sugerido, que el uso de estaciones de referencia podría facilitar la aplicación de estos métodos dentro de una misma ecoregión o ecotipo (Norris, 1995; Resh et al., 1995), y por tanto pueden ser válidas para la determinación de los límites de calidad. Así, en la segunda fase del GUADALMED será necesaria una ampliación del número de estaciones de referencia en cada tipo lo que permitirá la aplicación de análisis estadísticos con fiabilidad para desarrollar el método predictivo MEDPACS.

Otro problema añadido a la selección de condiciones de referencia y que normalmente no se tiene en cuenta es la presencia de especies alóctonas. A pesar de que un tramo de río pueda tener una muy buena calidad ecológica por lo que a los parámetros físico-químicos y algunos biológicos se refiere, la presencia de especies introducidas puede alterar las poblaciones de organismos (plantas o animales). Un problema importante de las cuencas mediterráneas es la gran proliferación del cangrejo de río americano (Procambarus clarkii), lo que puede suponer un cambio en la composición de macroinvertebrados y por tanto, afecta al índice biológico (Vila, com. pers.). En el caso de la vegetación de ribera, la presencia de especies vegetales alóctonas se incluye en el índice QBR (Munné et al., 1998), y se ha tenido en cuenta en los criterios de selección de las condiciones de referencia presentados en este artículo.

Una vez establecidas las condiciones de referencia para una zona, éstas deberían revisarse y cuestionarse cada cierto tiempo debido a un posible cambio de calidad de origen antrópico (cambio climático, cambio en los usos de la cuenca,...) (Owen et al., 2001). Además, una vez establecida esta red de referencia, los potenciales ecológicos de cada tipo deberán ser testados y modificados de nuevo.

Así pues, según los criterios establecidos, una estación sería considerada como de refe- rencia si la SCR es 10. En el caso que en un tipo no haya ninguna estación con puntuación 10, si el SCR está entre 7 y 9 (o 6 en algunos casos), todavía sería posible considerarla de referencia si lo que falla no es el \% cuenca natural, la regulación o la canalización. En este caso si lo que fallara fuera la físico-química, o el bosque de ribera, el problema se podría solucionar buscando un punto cercano con mejores condiciones (sin árboles alóctonos, con una mejor cobertura,...). Si en un tipo no hay ninguna estación con SCR 10, y todas las estaciones fallaran en el \% cuenca natural, la regulación y/o la canalización ( $\mathrm{Cal} / \mathrm{GB}$, por ejemplo) en lugar de hablar de referencia hablaríamos de MPE (máximo potencial ecológico) aceptando que estas 3 características son imposibles de cumplir y por tanto obviando 1,2 y/o 3 de los criterios, y calculando las estaciones MPE con el resto de criterios. Por lo tanto, las estaciones de los ecotipos de MPE deben tener un SCR igual o superior a 7. En el caso de que no se pueda (por ejemplo, en el ecotipo Llanura Aluvial), debido a que la físico-química o la ribera está alterada en un determinado punto, se debería tratar de hallar un punto cercano que cumpliera estas características de MPE. No obstante, si es imposible hallar tramos en mejores condiciones, se entiende que con medidas de restauración apropiadas se puede, como mínimo, conseguir el MPE.

\section{AGRADECIMIENTOS}

Este trabajo se ha realizado mediante la financiación de los proyectos HID98-0323-C05 y REN2001-3438-C07 del Ministerio de Ciencia y Tecnología y PLP/10/FS/97 de la Fundación Séneca de la CARM. Nuestro especial agradecimiento al Área de Coordinación y Aplicaciones Tecnológicas de la D.G.O.H. del Ministerio de Medio Ambiente, al Àrea de Medi Ambient de la Diputació de Barcelona, a la Delegación de Granada de la Consejería de Medio Ambiente de la Junta de Andalucía y a la Agencia Catalana de l'Aigua por su apoyo. 


\section{BIBLIOGRAFÍA}

ALBA-TERCEDOR, J. \& A. SÁNCHEZ-ORTEGA. 1988. Un método rápido y simple para evaluar la calidad biológica de las aguas corrientes basado en el de Hellawell (1978). Limnetica, 4: 51-56.

ALBA-TERCEDOR, J., P. JÁIMEZ-CUÉLLAR, M. ÁlVAREZ, J. AVILÉS, N. BONADA, J. CASAS, A. MELLADO, M. ORTEGA, I. PARDO, N. PRAT, M. RIERADEVALL, S. ROBLES, C. E. SÁINZ-CANTERO, A. SÁNCHEZ-ORTEGA, M.L. SUÁREZ, M. TORO, M. R. VIDALABARCA, S. VIVAS \& C. ZAMORA-MUÑOZ. 2002. Caracterización del estado ecológico de ríos mediterráneos ibéricos mediante el índice IBMWP (=BMWP'). Limnetica, 21: 175-185

BARBOUR, M. T. \& J. GERRITSEN. 1996. Subsampling of benthic samples: a defense of the fixed-count method. J. N. Am. Benthol. Soc., 15: 386-391.

BARBOUR, M. T., J. GERRITSEN, G. E. GRIFFITH, R. FRYDENBORG, E. MCCARRON, J. S. WHITE \& M. L. BASTIAN. 1996. A framework for biological criteria for Florida streams using benthic macroinvertebrates. J. N. Am. Benthol. Soc., 15: 185-211.

BARBOUR, M. T., J. GERRITSEN, B. D. SNYDER \& J. B. STRIBLING. 1999. Rapid Bioassessment Protocols for Use in Streams and Wadeable Rivers: Periphyton, Benthic Macroinvertebrates and Fish, Second Edition. Washington, D.C: EPA 841-B-99002. US EPA, Office of Water.

BENNION, H. \& J. FLUIN. 2001. Assessing changes in water quality and defining ecological reference conditions for a set of Scottish freshwater lochs, with reference to the EU Water Framework. $2^{\text {nd }}$ Symposium for European Freshwater Sciences, Toulouse, Francia: 12.

BONADA, N., N. PRAT, A. MUNNÉ, M. RIERADEVALL, J. ALBA-TERCEDOR, M. ÁlVAREZ, J. AVILÉS, J. CASAS, P. JÁIMEZCUÉllaR, A. MELLADO, G. MOYÀ, I. PARDO, S. ROBLES, G. RAMON, M. L. SUÁREZ, M. TORO, M. R. VIDAL-ABARCA, S. VIVAS \& C. ZAMORA-MUÑOZ. 2002. Ensayo de una tipología de las cuencas mediterráneas del proyecto GUADALMED siguiendo las directrices de la Directiva Marco del Agua. Limnetica, 21: 77-78.

CHOVAREC, A., P. JÄGER, M. JUNGWIRTH, V. KOLLER-KREIMEL, O. MOOG \& S. MUHAR.
2000. The Austrian way of assessing ecological integrity of running waters: a contribution to the EU Water Framework Directive. Hydrobiologia, 422/423: 445-452.

DAVIS, W. S. \& T. P. SIMON. 1995. Introduction. In: Biological assessment and criteria. Tools for water resource planning and decision making. W.S. Davis \& T.P. Simon (eds.): 3-6. Lewis Publishers, Boca Raton, Florida, USA.

D.O.C.E. 2000. Directiva 2000/60/CE del Parlamento Europeo y del Consejo de 23 de octubre de 2000 por la que se establece un marco comunitario de actuación en el ámbito de la politica de aguas. D.O.C.E. L 327 de 22.12.00. 69 pp.

EEKHOUT, S. E., C. A. BROWN \& J. M. KING. 1996. National Biomonitoring Programme for Riverine Ecosystems: Technical considerations and protocol for the selection of reference and monitoring sites. NBP Report Series $\mathrm{N}^{\circ} 3$. Pretoria, South Africa: Institute for Water Quality Studies. Department of Water Affairs and Forestry. $68 \mathrm{pp}$.

GERRITSEN, J. 1995. Additive biological indices for resource management. J. N. Am. Benthol. Soc., 14: 451-457.

GIBSON, G. M., M. T. BARBOUR, J. B. STRIBLING, J. GERRITSEN \& J. R. KARR. 1994. Biological criteria: technical guidance for streams. EPA/822-B-94-001. Washington, DC: Office of Science and Technology, US Environmental Protection Agency.

HUGHES, R. M. 1995. Defining acceptable conditions. In: Biological assessment and criteria: tools for water resource planning and decision making. W. S. Davis \& T. P. Simon (eds.): 31-48. Lewis Publishers, Boca Raton, Florida, USA.

INTERNATIONAL STANDARD ON WATER QUALITY. 2000. Biological classification in rivers, Part 1 (benthic macroinvertebrates) (EN ISO 8689-1:2000).

JÁIMEZ-CUÉLLAR, P., S. VIVAS, N. BONADA, S. ROBLES, A. MELLADO, M. ÁlVAREZ, J. AVILÉS, J. CASAS, M. ORTEGA, I. PARDO, N. PRAT, M. RIERADEVALL, C. E. SÁINZCANTERO, A. SÁNCHEZ-ORTEGA, M. L. SUÁREZ, M. TORO, M. R. VIDAL-ABARCA, C. ZAMORA-MUÑOZ \& J. ALBA-TERCEDOR. 2002. Protocolo GUADALMED (PRECE). Limnetica, 21: 187-204.

JOHNSON, R. K., T. WIEDERHOLM \& D.M. ROSENBERG. 1993. Freshwater biomonitoring 
using individual organisms, populations, and species assemblages of benthic macroinvertebrates. In: Freshwater biomonitoring and benthic macroinvertebrates. D. M. Rosenberg \& V. H. Resh (eds.): 40158. Chapman and Hall, New York, USA.

JOHNSON, R. K. 2001. Defining reference condition and setting class boundaries in ecological monitoring assessment. Background document for the EU-funded REFCOND project.

MELLADO, A., M. L. SUÁREZ, J. L. MORENO \& M. R. VIDAL-ABARCA. 2002. Aquatic macroinvertebrate biodiversity in the Segura River basin (S.E. Spain). Ver. Internat. Verein. Limnol., 28: 1-6.

MOSS, D. M., M. T. FURSE, J. F. WRIGTH \& P. D. ARMITAGE. 1987. The prediction of the macroinvertebrate fauna of unpolluted running-water sites in Great Britain using environmental data. Freshwat. Biol., 17:41-52.

MUNNÉ, A. \& N. PRAT. 1999. Regionalización de la cuenca del Ebro para el establecimiento de los objetivos del estado ecológico de sus ríos. Informe para la CHE (Oficina de Planificación Hidrológica). Zaragoza. 186 pp.

MUNNÉ, A., C. SOLÀ \& N. PRAT. 1998. QBR: Un índice rápido para la evaluación de la calidad de los ecosistemas de ribera. Tecnología del Agua, 175: 20-37.

MUNNÉ, A., N. PRAT, C. SOLÀ, N. BONADA \& M. RIERADEVALL. 2003. A simple field method for assessing the ecological quality of riparian habitat in rivers and streams. QBR index. Aquatic Conservation: Marine and Freshwater Ecosystems, 13: 147-164.

NORRIS, R. H. 1995. Biological monitoring: the dilemma of data analysis. J. N. Am. Benthol. Soc., 14: 440-450.

ORTEGA, M., M.L. SUÁREZ, M. R. VIDALABARCA, L. RAMÍREZ-DÍAZ. 1991. Aspectos dinámicos de la composición y estructura de la comunidad de invertebrados acuáticos de la Rambla del Moro después de una riada (Cuenca del Río Segura: SE. de España). Limnetica, 7: 11-24.

OWEN, R., W. DUNCAN \& P. POLLARD. 2001. Definition and establishment of reference conditions. Resumen reunión REFCOND. Ispra.

PARSONS, M. \& R. H. NORRIS. 1996. The effect of habitat-specific sampling of biological assessment of water quality using a predictive model. Freshwat. Biol., 36: 419-434.

PRAT, N. 1993. El futuro de los ríos españoles: secos o contaminados. Quercus, 84: 22-24
PRAT, N., A. MUNNÉ, C. SOLÀ, M. RIERADEVALL, N. BONADA \& G. CHACÓN. 2000. La qualitat ecològica del Llobregat, el Besòs i el Foix. Informe 1998. Estudis de la qualitat ecològica dels rius. Vol. 7. Barcelona: Diputació de Barcelona. Àrea de Medi Ambient.

PRAT, N., A. MUNNÉ, C. SOLÀ, M. RIERADEVALL, N. BONADA \& G. CHACON. 1999. La qualitat ecològica del Llobregat, el Besòs i el Foix. Informe 1997. Estudis de la qualitat ecològica dels rius. Vol. 6. Barcelona: Diputació de Barcelona. Àrea de Medi Ambient.

PRAT, N., A. MUNNÉ, N. BONADA, C. SOLÀ, M. PLANS, M. Rieradevall, R. CASANOVAS \& M. VILA. 2001. La qualitat ecològica del Llobregat, el Besòs, el Foix i la Tordera. Informe 1999. Estudis de la qualitat ecològica dels rius. Vol. 9. Barcelona: Diputació de Barcelona. Àrea de Medi Ambient.

RESH, V. H., R. H. NORRIS \& M. T. BARBOUR. 1995. Design and implementation of rapid assessment approaches for water resource monitoring using benthic macroinvertebrates. Aust. J. Ecol., 20: 108-121.

REYNOLDSON, T. B., R. C. BAILEY, K. E. DAY \& R. H. NORRIS. 1995. Biological guidelines for freshwater sediment based on Benthic Assessment of SedimenT (the BEAST) using a multivariate approach for predicting biological state. Aust. J. Ecol., 20: 285-294.

REYNOLDSON, T. B., R. H. NORRIS, V. H. RESH, K. E. DAY \& D. M. ROSENBERG. 1997. The reference condition: a comparison of multimetric and multivariate approaches to assess water-quality impairment using benthic macroinvertebrates. J. N. Am. Benthol. Soc., 16: 833-852.

ROBLES, S., M. TORO, C. NUÑO, J. AVILÉS, J. ALBA-TERCEDOR, M. ÁLVAREZ, N. BONADA, J. CASAS, P. JÁIMEZ-CUÉLLAR, A. MELLADO, A. MUNNÉ, I. PARDO, N. PRAT, M. L. SUÁREZ, M. R. VIDAL-ABARCA, S. VIVAS, G. MOYÁ \& G. RAMON. 2002. Descripción de las cuencas mediterráneas seleccionadas en el proyecto GUADALMED. Limnetica, 21: 35-61.

VIDAL-ABARCA, M. R., M.L. SUÁREZ, C. MONTES, A. MILLÁN, R. GÓMEZ, M. ORTEGA, J. VELASCO \& L. RAMÍREZ-DÍAZ. 1991. Estudio limnológico de la Cuenca del Río Mundo (Río Segura). Jornadas sobre el Medio Natural Albacetense: 339-357.

VV.AA. 1988. Estudio e interrelaciones de los niveles de calidad biológica y físico-química de las 
aguas de la Cuenca del Segura. Orígenes de la contaminación y bases para su planificación. Convenio ICONA-Universidad de Murcia. (Proyecto LUCDEME).

WRIGTH, J. F., D. MOSS, P. D. ARMITAGE \& M. T. FURSE. 1984. A preliminary classification of running-water sites in Great Britain based on macroinvertebrate species and the prediction of community type using environmental data. Freshwat. Biol., 14: 221-256.
WRIGHT, J.F. 1995. Development and use of a system for predicting the macroinvertebrate fauna in flowing waters. Aust. J. Ecol., 20: 181-197.

ZAMORA-MUÑOZ, C., C. E. SAINZ-CANTERO, A. SÁNCHEZ-ORTEGA \& J. ALBA-TERCEDOR. 1995. Are biological indices BMWP' and ASPT' and their significance regarding water quality seasonality dependent? Factors explaining their variations. Wat. Res., 29: 285290. 
Anexo 1. Valores de SCR de las estaciones muestradas, agrupadas según ecotipos (Bonada et al., en este volumen). Para la clave de las estaciones ver Robles et al., en este volumen. Los códigos con -R indican estaciones seleccionadas a priori por los expertos. Values of SCR for all sampling sites, grouped by ecotype (Bonada et al., in this issue). For a key of the sampling sites see Robles et al., in this issue. Codes with $-R$ show localities selected a priori by the expert panel.

\begin{tabular}{|c|c|c|c|c|c|c|c|c|}
\hline Tipo & Estación & SCR & Tipo & Estación & SCR & Tipo & Estación & SCR \\
\hline \multirow[t]{16}{*}{ Sil/Cab } & AD5-R & 10 & \multirow[t]{21}{*}{$\mathrm{Cal} / \mathrm{Cab}$} & B30 & 2 & \multirow[t]{45}{*}{$\mathrm{Cal} / \mathrm{MB}$} & AD2 & 3 \\
\hline & ALl & 6 & & GU2-R & 9 & & B12 & 5 \\
\hline & AL10 & 5 & & JU17 & 7 & & $\mathrm{~B} 22$ & 8 \\
\hline & ALl 1 & 7 & & JU1-R & 7 & & B25 & 5 \\
\hline & AL5 & 1 & & JU6 & 8 & & B28 & 8 \\
\hline & AL8 & 5 & & JU7-R & 7 & & GU3-R & 7 \\
\hline & B32-R & 9 & & JU8-R & 6 & & GU4 & 1 \\
\hline & B35-R & 10 & & L44-R & 10 & & JU15 & 4 \\
\hline & GU1 1 & 10 & & L56-R & 7 & & JU16 & 2 \\
\hline & GU12 & 5 & & MI10 & 7 & & JU19 & 5 \\
\hline & GU13 & 3 & & MI12-R & 7 & & JU2-R & 7 \\
\hline & GU14-R & 7 & & MI7 & 6 & & JU5 & 6 \\
\hline & GU15-R & 9 & & MI8-R & 7 & & L101 & 1 \\
\hline & GUl-R & 10 & & SEl-R & 10 & & L102 & 2 \\
\hline & GU5-R & 7 & & SE2-R & 10 & & L38 & 3 \\
\hline & GU6-R & 3 & & SE3-R & 9 & & L42 & 4 \\
\hline \multirow[t]{24}{*}{ Sil/MB } & $\mathrm{ADl}$ & 0 & & TU14 & 8 & & L54 & 10 \\
\hline & AD3-R & 4 & & TUl-R & 7 & & L60a-R & 8 \\
\hline & AD4-R & 3 & & TU4-R & 7 & & L60c & 6 \\
\hline & AL12 & 3 & & TU5 & 7 & & L61-R & 8 \\
\hline & AL13 & 1 & & TU6 & 8 & & L64a & 5 \\
\hline & AL14 & 4 & \multirow{15}{*}{ Temporales } & B24R & 10 & & L68 & 5 \\
\hline & AL15 & 6 & & SE4 & 9 & & L90 & 0 \\
\hline & AL16 & 3 & & TU12-R & 7 & & L94 & 1 \\
\hline & AL17 & 3 & & MIl & 7 & & MIl 1 & 6 \\
\hline & AL18 & 3 & & $\mathrm{P} 010$ & 5 & & MI4-R & 5 \\
\hline & AL2 & 5 & & P05 & 9 & & MI5 & 7 \\
\hline & AL3 & 3 & & P06 & 6 & & MI6 & 6 \\
\hline & AL4 & 2 & & S01 & 5 & & MI9 & 6 \\
\hline & AL9 & 1 & & S02 & 6 & & MI3 & 8 \\
\hline & $\mathrm{B} 10$ & 3 & & S03 & 3 & & PO3 & 1 \\
\hline & B15 & 3 & & S04 & 6 & & SE10-R & 6 \\
\hline & B16 & 2 & & S06 & 7 & & SE16-R & 8 \\
\hline & $\mathrm{B} 17 \mathrm{a}$ & 1 & & S07 & 9 & & SE 18-R & 9 \\
\hline & B4 & 3 & & B7a-R & 9 & & SE5-R & 8 \\
\hline & GU10-R & 1 & & B7-R & 8 & & SE6 & 3 \\
\hline & GU16 & 3 & \multicolumn{2}{|c|}{ LlanAluvial JU14 } & 2 & & SO5 & 1 \\
\hline & GU7-R & 1 & & JU18 & 4 & & TU10 & 5 \\
\hline & GU8 & 2 & \multirow[t]{15}{*}{ Ramblas } & AG3 & 4 & & TU7 & 4 \\
\hline & GU9 & 3 & & AG4 & 5 & & TU9-R & 7 \\
\hline \multirow[t]{13}{*}{ Karst } & AL6-R & 7 & & AG5 & 6 & & TU13 & 5 \\
\hline & AL7-R & 8 & & AG6 & 2 & & TU8 & 5 \\
\hline & AG7-R & 7 & & AG8 & 1 & & TU2 & 7 \\
\hline & AGl-R & 7 & & SEl 1 & 7 & & TU3 & 7 \\
\hline & AG2-R & 7 & & SE 12-R & 6 & & PO2 & 8 \\
\hline & PO9-R & 10 & & SE13 & 7 & \multirow[t]{8}{*}{ Cal/GB } & JU10 & 6 \\
\hline & PO11 & 10 & & SE14-R & 6 & & JU1 1-R & 5 \\
\hline & PO1-R & 10 & & SE15 & 7 & & JU12 & 6 \\
\hline & PO7-R & 10 & & SE17-R & 8 & & JU13 & 5 \\
\hline & PO8-R & 10 & & SE7 & 5 & & JU3 & 7 \\
\hline & L45R & 10 & & SE8-R & 5 & & JU4 & 6 \\
\hline & & & & SE9-R & 2 & & JU9 & 7 \\
\hline & & & & & & & TU1 1-R & 5 \\
\hline
\end{tabular}


Anexo 2. Estaciones muestreadas agrupadas según ecotipos, que cumplen los criterios de referencia o de Máximo Potencial Ecológico, o que podrían cumplirlos, según su SCR. Los códigos con $-\mathrm{R}$ indican estaciones que fueron seleccionadas a priori por los expertos. Sampling sites grouped by ecotype complying with criteria set out as reference conditions or as "Maximum Ecological Potential", or sites which could comply according to their SCR. Codes with-R are show sites selected a priori by the expert panel.

\begin{tabular}{|c|c|c|c|c|c|c|}
\hline & \multicolumn{4}{|c|}{ REFERENCIA } & \multicolumn{2}{|l|}{ MPE } \\
\hline & $\begin{array}{l}\text { Cumplen } \\
\text { criterios }\end{array}$ & SCR & $\begin{array}{l}\text { Podrían cumplirlos } \\
\text { fácilmente }\end{array}$ & SCR & $\begin{array}{l}\text { Cumplen } \\
\text { criterios }\end{array}$ & SCR \\
\hline \multirow[t]{4}{*}{ Sil/Cab } & AD5-R & 10 & B32 & 9 & & \\
\hline & B35-R & 10 & GU14-R & 7 & & \\
\hline & GU11 & 10 & & & & \\
\hline & GU1-R & 10 & & & & \\
\hline \multirow[t]{15}{*}{ Cal/Cab } & L44-R & 10 & GU2-R & 9 & & \\
\hline & SE1-R & 10 & GU3-R & 7 & & \\
\hline & SE2-R & 10 & JU1-R & 7 & & \\
\hline & & & JU6 & 8 & & \\
\hline & & & JU7-R & 7 & & \\
\hline & & & JU8-R & 6 & & \\
\hline & & & L56-R & 7 & & \\
\hline & & & MI12-R & 7 & & \\
\hline & & & MI7 & 6 & & \\
\hline & & & MI8-R & 7 & & \\
\hline & & & SE3-R & 9 & & \\
\hline & & & TU14 & 8 & & \\
\hline & & & TU1-R & 7 & & \\
\hline & & & TU4-R & 7 & & \\
\hline & & & TU6 & 8 & & \\
\hline \multirow{8}{*}{ Temporales } & B24-R & 10 & SE4 & 9 & & \\
\hline & & & TU12-R & 7 & & \\
\hline & & & MI1 & 7 & & \\
\hline & & & B7a-R & 9 & & \\
\hline & & & B7-R & 8 & & \\
\hline & & & PO5 & 9 & & \\
\hline & & & $\mathrm{SO} 7$ & 9 & & \\
\hline & & & SO6 & 7 & & \\
\hline \multirow[t]{6}{*}{ Karst } & L45-R & 10 & AL6-R & 7 & & \\
\hline & PO9-R & 10 & AL7-R & 8 & & \\
\hline & PO11 & 10 & AG7-R & 7 & & \\
\hline & PO1-R & 10 & AG1-R & 7 & & \\
\hline & PO7-R & 10 & AG2-R & 7 & & \\
\hline & PO8-R & 10 & & & & \\
\hline \multirow[t]{5}{*}{$\mathrm{Cal} / \mathrm{MB}$} & L54 & 10 & B22 & 8 & & \\
\hline & & & JU2-R & 7 & & \\
\hline & & & MI3 & 8 & & \\
\hline & & & SE5-R & 8 & & \\
\hline & & & PO2 & 8 & & \\
\hline \multirow[t]{2}{*}{ Cal/GB } & & & & & JU3 & 7 \\
\hline & & & & & JU9 & 7 \\
\hline Ramblas & & & SE15 & 7 & & \\
\hline
\end{tabular}

